\title{
Hacia una traducción de "The Double Image", de Anne Sexton: apuntes sobre la bidireccionalidad de la maternidad
}

Víctor Anguita-Martínez

$\mathrm{V}$ iktor Frankl, ilustre psicólogo y miembro de la denominada tercera escuela vienesa de psicoterapia, afirmaba que "realmente no existen enfermos 'del espíritu', pues el espíritu, la persona espiritual misma, no puede enfermarse, y permanece allí, detrás de la psicosis, aun cuando la mira del psiquiatra apenas la puede distinguir" (2012: 108). Es decir, el paciente de salud mental, bajo ningún concepto, pierde su esencia, y no es esta la que hay que sanar, sino su sintomatología patológica.

Este es el caso de la bostoniana Anne Sexton (1928-1974), galardonada con el premio Pulitzer de poesía en 1967 por Live or Die (1966) y representante del movimiento confesional poético, que sentó las bases para la concepción de una lírica innovadora en su contexto; en esta corriente literaria la acompañan Robert Lowell y Sylvia Plath. La poesía confesional se caracteriza principalmente por su temática personal, cuya voz poética la componen una serie de personae, a caballo entre la realidad y la exaltación de las experiencias pasadas, proyectadas en un alter ego expuesto al estudio público. A tal respecto, traemos a colación las palabras de Salvio, que ilustran la utilidad de la construcción de estas proyecciones: 
One lesson offered to us by Anne Sexton, however, is that the melancholic can work at using the art of composing personae to restage the effort to remember her loss, thereby gaining insight into how loss can acquire meaning, and potentially generate recovery, not of the bereaved, but of herself, as the person who remembers (2007: 54).

Además del estilo y los temas que aborda su poesía, considerados tabú para la burguesía estadounidense de mediados de siglo, lo más peculiar de la vida y legado de Sexton es el origen de su prolífera obra, si tenemos en cuenta el corto abanico de tiempo con el que contó. Fue diagnosticada con una depresión posparto que derivó en un trastorno bipolar con tendencias suicidas. El estado maniaco-depresivo marcó su vida no solo de forma negativa, sino que la llevó a conocer al Dr. Orne, que la animó a escribir como parte de un tratamiento psicoanalítico innovador. Por este motivo, las voces de la manía, la decadencia recogida entre los muros del centro psiquiátrico y la contemplación del suicidio ocupan el lugar central de sus versos. Y es precisamente de esas anotaciones terapéuticas y de la participación en talleres de poesía impartidos por John Holmes y Lowell que se forja To Bedlam and Part Way Back (1963), donde profundiza en "themes of loss and mourning and attest to the psychic and social threat of cancer, early sexual distress, addictions, and madness” (Salvio, 2007: 21).

\section{OBJETO DE ESTUdio}

Con el presente estudio pretendemos ofrecer una traducción actualizada del poema "The Double Image”, incluido en To Bedlam and Part Way Back (1963). Se trata esta última de su obra debut y, por los motivos expuestos líneas arriba, lo particular de su nacimiento se refleja en una condensación de poemas de índole biográfica que giran en torno a las entradas y salidas de la autora del centro psiquiátrico y al rol social de la mujer en el que no se terminaba de encontrar.

De este poemario circula una traducción en el mercado editorial español: la obra antológica Anne Sexton. Poesía completa (2012), de José Luis Reina Palazón, publicada por la editorial Linteo. ${ }^{1}$ Gracias a la confrontación entre distintos poemas en lengua original y en español, hemos podido comprobar la necesidad de un cambio de estrategia respecto a la traducción literal por la que se decanta el traductor, ya que esta parece ser limitante en la recreación de los efectos estilísticos de los versos sextonianos. No podemos perder de vista el cambio de paradigma en los factores rítmicos y métricos de la poesía contemporánea, también recogidos en la obra que aquí tratamos:

La especie poemática, en el [...] presente no se aviene a ser definida en virtud de parámetros computables, evidentes a simple vista, y frecuentemente ni tan siquiera conceptualizables de manera estable. Hace ya tiempo que la métrica, la acentuación o la rima no son criterios de poeticidad, o, por lo menos, no son los únicos, con lo que no se les puede invocar como criterios decisorios absolutos de la naturaleza poética de un texto. E incluso las cuestiones de musicalidad y ritmo — tan solicitadas por la poesía moderna una vez que se rompió el molde poético clásico — tampoco reciben hoy título de criterio poético básico (Gamoneda Lanza, 2008: 42-43)

1 Por cuestiones de espacio y porque escapa a las intenciones de este trabajo, no analizamos esta obra en cuestión, pero remitimos a estudios precedentes en cuestiones traductológicas (Anguita Martínez, 2019; 2020a; 2020b). 
Este acercamiento lingüístico y formal, sin embargo, no es funcional a la hora de traspasar la poesía de la bostoniana debido a la importancia que esta otorga a la concatenación de metáforas y símiles. Consideramos más pertinente la transcreación de Etkind (1982) por su postura conciliadora entre la forma y el contenido del texto original. Dicha prioridad a la conservación del sentido sin que la forma (en este caso, los referentes metafóricos) se vea alterada, es posible recurriendo a la pragmática. Sobre ello, tomamos como base los estudios de Fouces González (2007), Ramírez García (2009), y Samaniego Fernández (2009), cuyas aportaciones nos han servido como referencia a la hora de intentar exportar los efectos del poema inglés al español.

\section{"The Double Image": tríPtico RELACIONAL}

En To Bedlam and Part Way Back (1960), el despliegue de hechos biográficos descritos con el estilo confesional de Anne Sexton nos permite acercarnos a su intimidad emocional y a los callejones sin salida de su patología. La difícil relación que mantenía con su madre y sus hijas fue, sin lugar a dudas, la roca atada a los pies que le impidió salir a flote de la depresión en la que se ahogaba. En “The Double Image", la poeta entrelaza dos roles vitales, su yo madre y su yo hija, con su yo enferma-suicida. Esta trenza situacional es una carta en el tiempo a su descendiente, una justificación y un reclamo de perdón por la ausencia durante sus primeros años de vida. Constituye una exposición del cargo de conciencia, de su frustración como madre y como hija, consecuencia de su fragilidad y juego con la muerte: “The poem describes, among other things, Sexton's estrangements from her baby daughter, her suicide attemps and hospitalizations, and her mother's death from cancer" (Javadizadeh, 2016: 77).

En cuanto a la composición del poema, y sin pretender extendernos demasiado, intercambió cartas con su círculo en donde exponía abiertamente la forma en la que lo había trabajado y pedía ayuda crítica para terminar de pulirlo:

[Carta dirigida a Snodgrass]: Aquí tienes el poema, terminado por el momento. Al parecer mi pequeña escapada al 'hotel blindado' me ayudó a desbloquear el proceso de su escritura. Solo quiero saber si tú crees que funciona, si tiene un motivo su violencia, un motivo para ser escrito (o más bien leído) más allá de mi propia necesidad de darle forma al caos. [...] Devuélveme esto cuando termines, salvo que quieras quedártelo porque es un fastidio copiarlo a máquina. Solo hay ciertas partes del poema donde cuento las sílabas - las otras son más libres, a pesar de lo que pueda parecer a primera vista (Anne Sexton, en Gray, 2015: 82).

[Carta dirigida a Nolan Miller]: Hoy, como es Acción de Gracias, doy gracias por haber terminado mi poema 'largo'. No te he escrito antes por culpa del maldito poema y porque quiero tu opinión y tus críticas. Te envío los otros (para que veas que sigo escribiendo) y por si quieres comentar alguna cosa, pero el que realmente me importa es «La doble imagen». Es una especie de voz; ipero es una buena voz? Ya sabes, Nolan, que la gente me repite una y otra vez que no he encontrado mi 'voz' aún y he dedicado bastante tiempo a rebuscar en mi escritorio y bajo viejos manuscritos y no he encontrado ningún nuevo sonido destacable. Así que escribí este largo poema sobre mis mejores folios como lo mereciera desde el principio (Anne Sexton, en Gray, 2015: 85). 
Sexton no solo contó con la aprobación y la acogida del público, sino con la de su madre, que tuvo ocasión de leer el borrador poco antes de fallecer: "Mary Gray había visto el manuscrito de "The Double Image" antes de ingresar en el hospital y dijo que le gustaba" (Middlebrook, 1998: 18). Con el beneplácito de su progenitora, el poema fue publicado en la prestigiosa revista literaria The Hudson y, posteriormente, en el penúltimo lugar de su debut editorial.

La relación materno-filial bidireccional que aquí tratamos no es una temática exclusiva del texto. La institucionalización del manicomio y la enfermedad mental (Javadizadeh, 2016: 88-89) se relaciona con los símiles entre el psiquiátrico, el hospital y la universidad: "the hospital is like the university in that both are disciplinary institutions, whose profesional staffs are empowered [...] with policing the subject into conformity with their norms and standars". Asimismo, el poema refleja el interés de la autora por la conservación de fotografías y retratos (Helle, 2016), lo que a su vez expresa su duelo recurrente por aquellos familiares que la abandonaron casi contemporáneamente.

\title{
PROPUESTA DE TRADUCCIÓN
}

A continuación, proponemos una traducción actualizada cuya máxima pretensión es recrear los efectos del poema original sin que esto suponga la pérdida del estilo característico de Sexton, marcada por una contextualización temporal remarcada (el suceder de los meses) y sin alterar el equilibrio entre el contenido explícito e implícito tras las figuras retóricas. Con respecto a la escansión métrica, nos decantamos por el verso libre, motivados por lo expuesto hasta ahora y por la confesión de la propia autora sobre la composición de este poema en el que "[s]olo hay ciertas partes donde cuento las sílabas - las otras son más libres, a pesar de lo que pueda parecer a primera vista” (Anne Sexton, en Gray, 2015: 82)

\author{
The Double Image ${ }^{2}$ \\ LA DOBLE IMAGEN
}

1.

I am thirty this November.

You are still small, in your fourth year. We stand watching the yellow leaves go queer,

flapping in the winter rain.

falling flat and washed. And I remember mostly the three autumns you did not live here.

They said I'd never get you back again.

I tell you what you'll never really know:
1.

Yo cumplo treinta el próximo noviembre. Tú todavía eres pequeña con tus cuatro años. Juntas contemplamos el remolino de hojas

[amarillas

ondeando en la lluvia invernal cayendo apaisadas y lavadas. Y recuerdo sobre todo los tres otoños que no viviste aquí. Me dijeron que nunca te recuperaría. Te diré algo de lo que jamás tendrás la certeza:

2 Sexton, Anne (1960), "The Double Image", en To Bedlam and Part Way Back, Boston, Houghton Mifflin. 
all the medical hypothesis

that explained my brain will never be as true as

[these

struck leaves letting go.

I, who chose two times

to kill myself, had said your nickname

the mewling mouths when you first came;

until a fever rattled

in your throat and I moved like a pantomine above your head. Ugly angels spoke to me. The

[blame,

I heard them say, was mine. They tattled like green witches in my head, letting doom

leak like a broken faucet;

as if doom had flooded my belly and filled your

[bassinet,

an old debt I must assume.

Death was simpler than I'd thought.

The day life made you well and whole

I let the witches take away my guilty soul.

I pretended I was dead

until the white men pumped the poison out,

putting me armless and washed through the

[rigamarole

of talking boxes and the electric bed.

I laughed to see the private iron in that hotel.

Today the yellow leaves

go queer. You ask me where they go I say today

[believed

in itself, or else it fell.

Today, my small child, Joyce,

love your self's self where it lives.

There is no special God to refer to; or if there is, todas las hipótesis médicas

que explicaban mi cerebro nunca serán más

[veraces que

esas hojas desplomadas tras dejarse caer.

Yo, que decidí dos veces

suicidarme, pronuncié tu apodo

en los meses de maullidos cuando llegaste;

hasta que una fiebre carraspeó

en tu cuello y yo actué como en una pantomima sobre tu cabeza. Ángeles feos me hablaron.

[La culpa,

los oí decir, era mía. Chismorreaban

como brujas verdes en mi cabeza, dejaban que

[la ruina

gotease como un grifo que pierde;

como si la ruina hubiera anegado mi vientre y

[desbordado tu moisés,

una vieja deuda que debo asumir.

La muerte era más simple de lo que pensaba.

El día en que la vida te trajo sana y bien

permití a las brujas que se llevaran mi alma

[culpable.

Fingí estar muerta

hasta que los hombres blancos me extirparon

[el veneno

y me entregaron sin brazos y limpia al runrún

de los altavoces y la cama eléctrica.

Reí al ver los grilletes privados de aquel hotel.

Hoy las hojas amarillas

remolinean. Me preguntas a dónde van y yo te

[digo que el hoy cree

en sí mismo, si no se derrumbaría.

Hoy, mi pequeña, Joyce,

ama al ser de tu propio ser allá donde viva.

No existe un Dios especial al que dirigirse; o si

[lo hay, 
why did I let you grow

in another place. You did not know my voice when I came back to call. All the superlatives

of tomorrow's white tree and mistletoe

will not help you know the holidays you had to

[miss.

The time I did not love

myself, I visited your shoveled walks; you held

[my glove.

There was new snow after this.

2.

They sent me letters with news

of you and I made moccasins that I would never

[use.

When I grew well enough to tolerate myself, I lived with my mother. Too late, too late, to live with your mother, the witches

[said.

But I didn't leave. I had my portrait done instead.

Part way back from Bedlam

I came to my mother's house in Gloucester,

Massachusetts. And this is how I came

to catch at her; and this is how I lost her.

I cannot forgive your suicide, my mother said.

And she never could. She had my portrait

done instead.

I lived like an angry guest,

like a partly mended thing, an outgrown child.

I remember my mother did her best.

She took me to Boston and had my hair restyled. por qué te dejé crecer

en otro lugar. No conocías mi voz

cuando irrumpía en tu vida. Todos los

[superlativos

del árbol blanco del mañana y el muérdago

no te ayudarán a descubrir las vacaciones que te

[perdiste.

El tiempo en que no me quería

a mí misma, visité tus caminos espalados; me

[cogiste del guante.

Después llegó nieve naciente.

2.

Me enviaban cartas con noticias

tuyas y hacía mocasines que nunca me ponía.

Cuando mejoré lo suficiente como para tolerarme

a mí misma, viví con mi madre. Demasiado tarde,

demasiado tarde, para vivir con tu madre,

[dijeron las brujas.

Pero no me fui. En cambio

mandé pintar mi retrato.

A medio camino al regresar de Manicomio me llegué a casa de mi madre en Gloucester,

Massachusetts. Y así fue como llegué para aferrarme a ella; y así fue como la perdí. No puedo perdonar tu suicidio, dijo mi madre. Y nunca pudo. En cambio mandó pintar mi retrato.

Viví como un invitado furioso, como un remiendo a la mitad, una niña tras el [estirón.

Recuerdo que mi madre hizo cuanto pudo. Me llevó a Boston para cambiarme el corte de

[pelo. 
Your smile is like your mother's, the artist said.

I didn't seem to care. I had my portrait done instead.

There was a church where I grew up with its white cupboards where they locked us

[up,

row by row, like puritans or shipmates singing together. My father passed the plate.

Too late to be forgiven now, the witches said.

I wasn't exactly forgiven. They had my portrait done instead.

3.

All that summer sprinklers arched over the seaside grass.

We talked of drought while the salt-parched

field grew sweet again. To help time pass

I tried to mow the lawn and in the morning I had my portrait done, holding my smile in place, till it grew formal.

Once I mailed you a picture of a rabbit and a postcard of Motif number one, as if it were normal to be a mother and be gone.

They hung my portrait in the chill north light, matching me to keep me well. Only my mother grew ill.
Tienes la misma sonrisa que tu madre, dijo el [pintor.

Me fue indiferente. En cambio mandé pintar mi retrato.

Había una iglesia donde me crié con armarios blancos donde nos encerraban

fila a fila, como puritanos o tripulantes que cantan al unísono. Mi padre pasaba la

[canastilla.

Demasiado tarde para el perdón, dijeron las

[brujas.

Nunca me llegaron a perdonar. En cambio mandaron pintar mi retrato.

3.

Todo aquel verano los aspersores se arquearon sobre la hierba de la orilla.

Hablamos de la sequía mientras el sediento campo salinizado se endulzaba de nuevo. Para matar el [tiempo

intentaba cortar el césped y en las mañanas mandaba pintar mi retrato, contuve la sonrisa en su sitio, hasta que quedó

[fija.

$$
\begin{gathered}
\text { Una vez te mandé el dibujo de un conejo } \\
\text { y una tarjeta con el número uno diseñado } \\
\text { como si fuera normal } \\
\text { ser madre y ser ausencia. } \\
\text { Colgaron mi retrato en la fría } \\
\text { luz del norte, para retarme } \\
\text { al reflejo en ese bienestar. }
\end{gathered}
$$

Fue mi madre quien cayó enferma. 
She turned from me, as if death were catching,

as if death transferred, as if my dying had eaten inside of her. That August you were two, by I timed my days [with doubt.

On the first of September she looked at me and said I gave her cancer.

They carved her sweet hills out and still I couldn't answer.

4.

That winter she came part way back from her sterile suite of doctors, the seasick cruise of the X-ray, the cells' arithmetic gone wild. Surgery incomplete, the fat arm, the prognosis poor, I heard them say.

During the sea blizzards she had here own portrait painted. A cave of mirror placed on the south wall; matching smile, matching contour.

And you resembled me; unacquainted with my face, you wore it. But you were mine after all.

I wintered in Boston, childless bride, nothing sweet to spare with witches at my side. I missed your babyhood, tried a second suicide, tried the sealed hotel a second year.
Me dio la espalda, como si la muerte estuviera

[de caza

como si la muerte fuera trasferible, como si mi morir la hubiera devorado por dentro.

Aquel agosto tú tenías dos años, aunque yo [contaba insegura los días.

El uno de septiembre me miró y me dijo que le había provocado un cáncer. Le socavaron sus dulces montañas y aún entonces no supe responder.

4.

Aquel invierno ella hizo

la mitad del camino de vuelta de su suite esterilizada de doctores, el vahído en el crucero de los rayos X, la aritmética de las células desenfrenadas. La cirugía incompleta, el brazo abultado, la prognosis pobre, los oí decir.

Durante el mar de nevascas ella encargó pintar su propio retrato. Una caverna en espejo colgada de la pared sur; sonrisa idéntica, silueta idéntica. Y te parecías a mí; estabas poco familiarizada con mi cara para vestirla. Pero tú me pertenecías a fin de cuentas.

Inverné en Boston, esposa sin hijos, sin nada dulce que repartir entre las brujas aquí a mi lado.

Me perdí tu niñez, probé un segundo suicidio, probé el hotel hermético un segundo año. 
On April Fool you fooled me. We laughed and

this

was good.

5.

I checked out for the last time

on the first of May;

graduate of the mental cases,

with my analysts's okay,

my complete book of rhymes,

my typewriter and my suitcases.

All that summer I learned life back into my own

seven rooms, visited the swan boats,

the market, answered the phone,

served cocktails as a wife

should, made love among my petticoats

and August tan. And you came each weekend. But I lie.

You seldom came. I just pretended you, small piglet, butterfly

girl with jelly bean cheeks, disobedient three, my splendid

stranger. And I had to learn why I would rather die than love, how your innocence would hurt and how I gather

guilt like a young intern his symptons, his certain evidence.

That October day we went

to Gloucester the red hills

reminded me of the dry red fur fox

coat I played in as a child; stock still

like a bear or a tent,

like a great cave laughing or a red fur fox.
El día de los Inocentes me gastaste la

[inocentada. Reímos

y eso fue algo bueno.

5.

Dejé el hotel por última vez

el primer día de mayo;

graduada en tarados,

con la aprobación de mi terapeuta,

mi libro de rimas completo,

mi máquina de escribir y mis maletas.

Durante ese verano aprendí a vivir de nuevo en mis propias

siete estancias, visité las barcas de cisne,

el supermercado, respondí al teléfono, serví copas como una esposa

ha de hacer, hice el amor entre mis enaguas

y el bronceado agostizo. Y tú venías todos

los fines de semana. Pero miento.

Apenas venías. Solo fingía

tu presencia, cerdita mía, niña

mariposa con grajeas en los mofletes,

tres años de desobediencia, mi espléndida

desconocida. Y tuve que aprender por qué anteponía

la muerte al amor, por qué dolía

tu inocencia y por qué recolectaba

culpa cual médico residente

sus síntomas, su prueba certera.

Aquel día de octubre en que fuimos

a Gloucester las montañas rojizas

me recordaron el abrigo de piel reseca

de zorro rojo que lucía de niña; todavía pendido como un oso o una tienda,

como una gigantesca cueva sonriente o un zorro 
We drove past the hatchery,

the hut that sells bait,

past Pigeon Cove, past the Yacht Club, past

[Squall's

Hill, to the house that waits

still, on the top of the sea,

and two portraits hung on the opposite walls.

6.

In north light, my smile is held in place, the shadow marks my bone. orchid; my mocking mirror, my overthrown love, my first image. She eyes me from that face

that stony head of death I had outgrown.

The artist caught us at the turning;
Pasamos por el vivero,

la cabaña donde venden cebos, dejando atrás Pigeon Cove, Yacht Club, Squall's

Hill, en dirección a la casa que esperando sigue, sobre el mar, y a los dos retratos colgados en paredes [enfrentadas.

En la luz del norte, mi sonrisa sigue en su sitio, la sombra me resalta los huesos. Con qué estaría soñando mientras estaba ahí

[sentada,

toda yo con la espera en los ojos, el lugar de la sonrisa, el rostro joven, el cepo para zorros.

En la luz del sur, su sonrisa sigue en su sitio, sus mejillas se marchitan como una orquídea seca; mi espejo socarrón, mi amor

we smiled in our canvas home

before we chose our foreknown separate ways.

The dry red fur fox coat was made for burning.

I rot on the wall, my own

Dorian Gray.

And this was the cave of the mirror, that double woman who stares at herself, as if she were petrified esa cabeza insensible de la muerte que se me había quedado pequeña.

El artista nos captó giradas; sonreímos en nuestro lienzo hogar antes de seguir nuestra hoja de ruta por [caminos distintos.

El abrigo de piel reseca de zorro rojo nació para

[arder.

Me pudro en la pared, mi propio

Dorian Gray.

Y esta es la caverna en espejo, esa mujer doble que fija la mirada en sí misma, como si se hubiera petrificado 
in time - two ladies sitting in umber chairs.

You kissed your grandmother and she cried.

7.

I could not get you back

except for weekends. You came

each time, clutching the picture of a rabbit

that I had sent you. For the last time I unpack

your things. We touch from habit.

The first visit you asked my name.

Now you stay for good. I will forget how we bumped away from each other like

[marionettes

on strings. It wasn't the same

as love, letting weekends contain

us. You scrape your knee. You learn my name,

wobbling up the sidewalk, calling and crying.

You can call me mother and I remember my

[mother again,

somewhere in greater Boston, dying.

I remember we named you Joyce

so we could call you Joy.

You came like an awkward guest

that first time, all wrapped and moist

and strange at my heavy breast.

I needed you. I didn't want a boy,

only a girl, a small milky mouse

of a girl, already loved, already loud in the house

of herself. We named you Joy.

I, who was never quite sure

about being a girl, needed another

life, another image to remind me. en el tiempo - dos señoras sentadas en sillo-

[nes ocre.

Tú le diste un beso a tu abuela

y ella lloró.

\section{7.}

No podía recuperarte

salvo los fines de semana. Venías

siempre, apretando la imagen del conejo

que te envíe. Por última vez desembalo

tus cosas. Nos tocamos por costumbre.

En tu primera visita me preguntaste mi nombre. Ahora has venido para quedarte. Olvidaré que nos distanciábamos a saltitos como

[marionetas

en las cuerdas. No era lo mismo

que amar, el dejar que nos sumaran los fines de semana. Te raspas la rodilla. Aprendes mi

[nombre,

mientras te tambaleas por la acera, gritas y lloras.

Sabes llamarme madre y así yo recuerdo a mi

[madre,

en algún lugar del gran Boston, muriéndose.

Recuerdo que te llamamos Joyce

para nombrarte nuestra Alegría.

Llegaste como un huésped incómodo

aquella primera vez, toda envuelta y húmeda

y extraña a mi pecho pesado.

Te necesitaba. No quería un niño,

solo una niña, una ratoncita

presumida, ya querida, ya ruidosa desde la casa de sí misma. Te nombramos nuestra Alegría.

Yo, que nunca tuve la certeza

de ser una niña, necesitaba otra

vida, otra imagen para recordarme. 
And this was my worst guilt; you could not cure

or soothe it. I made you to find me.
Y esta fue la mayor de mis culpas; tú no

[pudiste curarlo

o apaciguarlo. Te hice para encontrarme.

\section{ANÁLISIS TRADUCTOLÓGICO}

Detenernos en un estudio filológico en términos estilísticos y exponer el contenido biográfico a lo largo del poema nos ocuparía un estudio individual. Anne Sexton cuenta al lector las visitas reguladas de la pequeña Joyce, cuando todavía eran unas desconocidas que compartían la rutina del afecto y que ahora han aprendido a convivir; su salida del centro psiquiátrico y la dificultad para readaptarse a la vida fuera de la institución; los cuidados de su madre que, a mitad del poema, se invierten para ser ella quien cuide de su progenitora hasta su muerte. También expone el anhelo por el perdón que no recibe, el peso de su condición mental para su familia y los vínculos relacionales entre ellos hasta hacer un viaje real y en el tiempo de regreso a la casa de su madre para una última despedida.

En coherencia con nuestro objetivo traductor y dando preferencia al contenido metafórico del texto original en inglés, hemos intentado mantener los referentes en la medida de lo posible. Esto no significa que no se haya considerado la sonoridad del original en la reescritura en lengua española. Las ocasiones en las que la autora ha manifestado una rima voluntaria, cuando la conservación semántica no lo ha permitido, las hemos compensado en otros versos, como "En cambio / mandé pintar mi retrato", truncamiento que en inglés no rima y en cuya rima española nos apoyamos para recuperar el recurso rítmico.

Las estrategias para reconstruir la fonoestilística del poema no se han adoptado exclusivamente en la rima final de verso. La repetición de sonidos no fue ignorada en la traducción, por ello, el verso final de estrofa "There was new snow after this", donde Anne Sexton recrea el silbido de la ventisca que trae consigo la nevada mediante la alternancia de 's' y ' $w$ ', lo hemos trasladado en español como "Después llegó nieve naciente". Esta redundancia de la 'n' y el diptongo 'ie' nos ha permitido mantener el efecto sin alterar el sentido del Texto Origen (TO). Otro ejemplo de este fenómeno sonoro es la compensación de la rima interna "and strange at my heavy breast" por la repetición de sonidos "y extraña a mi pecho pesado".

Con idéntica intención nos decantamos por una transposición en los siguientes casos: en primer lugar, traducimos "as if death were catching / as if death transferred, / as if my dying had eaten inside of her", por "como si la muerte estuviera de caza / como si la muerte fuera trasferible, / como si mi morir la hubiera devorado por dentro", lo cual nos ayuda a mantener el paralelismo estructural y anafórico. En segundo lugar, la paradoja "as if it were normal / to be a mother and be gone", con la que expone su sentimiento de culpa porque su hija, acostumbrada a no tenerla cerca, tolera su ausencia, se consigue en inglés porque las estructuras 'to be + adjetivo' y 'voz pasiva + participio' lo hacen posible. En español, esta voz pasiva no es normativa y el auxiliar correcto es 'haber' en su forma reflexiva. Para no perder el juego de palabras y la anáfora interversal, repetimos la técnica de la transposición para remitir al verbo 'ser': "como si fuera normal / ser madre y ser ausencia”.

Para no perder el estilo de la autora, la sintaxis tendencialmente breve que provoca en el lector un efecto de ráfagas de recuerdo y el comentario crítico de su sentimiento hacia esto con la falta de 
conectores discursivos y una densa adjetivación, hemos optado por opciones que alzan el registro, como "bronceado agostizo"; la transposición gramatical "por qué anteponía / la muerte al amor" de "why I would rather / die than love"; y el uso menos extendido del verbo 'probar' en el paralelismo "probé con un segundo suicidio, / probé el hotel hermético un segundo año”. En inglés, el verbo 'try' aúna el verbo 'intentar' y 'experimentar', por lo que el recurso fonético no eleva el registro, mientras que con nuestra reformulación se altera ligeramente en virtud de la figura retórica.

Centrándonos en los límites simbólicos y semióticos de la combinación lingüística que aquí entran en juego, encontramos dos retos de traducción de especial interés que ponen en peligro la carga metafórica e implícita del original. Hemos intentado reducir la pérdida intríseca a la limitación lingüística "On April Fool you fooled me". En Estados Unidos, el Día de los Santos Inocentes tiene lugar en el mes de abril y, por eso, la poeta se divierte con la inocentada que su hija le gasta. Pero este retruécano no evoca casualmente un momento con Joyce entre bromas, sino que lleva el sello de la enfermedad mental en las implicaturas de la onomástica en el calendario. Una persona 'fool' carece de juicio, de razón. Anne Sexton remite a su locura y al modo en que su hija es consciente de ella, pues consigue engañarla y demostrar un ingenio superior, cuando normalmente son los padres quienes timan a pequeña escala a sus hijos con juegos como el hurto de la nariz. Por lo tanto, la elección de esta fecha no es casual, y de ella el lector recupera por inferencias el contenido para concluir la perfecta descodificación del mensaje tras los malabares poéticos.

A la hora de traducir, partimos de la base de que el Día de los Santos Inocentes, en España, tiene lugar el 28 de diciembre. Es cierto que la cronología en los versos es crucial, ya que el mes de abril enlaza el invierno en Boston con su salida del psiquiátrico en mayo, que introduce en la parte 5 del poema, pero premia el sentido y la cohesión por encima de ese recurso. Es inevitable perder las implicaturas con el término 'fool' y su estado de enajenación por la nomenclatura de esa fecha en el calendario. Hemos optado por adaptar el referente a su equivalente en nuestra cultura, aunque signifique el cambio temporal. Esto nos ha permitido conservar también la anáfora del original y la relación con el éxito de la broma de su hija hacia ella.

Una pérdida importante y, a nuestro juicio, inevitable, es el cierre del poema. En la séptima parte, la escritora concentra toda la atención en su hija y llena de ternura la estrofa. Concluye el mensaje con el inicio de todo, la motivación que la llevó a quedarse embarazada. Destaca la belleza del juego de palabras en la elección del nombre de la pequeña, "I remember we named you Joyce / so we could call you Joy”, con el que remite a la 'alegría' que espera que su llegada traiga a la familia, una idea clave que el lector capta como primordial en la reiteración posterior unos versos después, "We named you Joyce”. La voz poética empieza a confesar la esperanza puesta en el nacimiento de una niña para sacarla del estado depresivo en el que se encuentra y llenar todo de color.

La traducción de este juego de Anne Sexton con el diminutivo del nombre de su hija 'JoyceJoy' no es trasladable porque no comparten significante. Es por eso que la traducción pierde ese contenido implícito reforzado en la anáfora y que permite al lector angloparlante gozar del ingenio de la poeta. En "Recuerdo que te llamamos Joyce / para nombrarte nuestra Alegría", hemos optado por el nombramiento de la dicha familiar para compensar el juego de palabras, una explicitación del símil que aparece reforzado unos versos más abajo con el eco anafórico "Te nombramos nuestra Alegría". Además, recurrimos a la disposición gráfica del poema convirtiendo ese sentimiento en una alegoría personalizada con la mayúscula. Si bien somos conscientes de la pérdida, consideramos que con esta opción el lector puede percibir el hincapié en la felicidad con la que Anne Sexton vivió la llegada de la pequeña Joyce a su vida. En cualquier caso, hemos recuperado los efectos estilísticos con la 
nominalización de verbos y la conservación de la adjetivación y la sintaxis del original, de modo que el lector meta pueda disfrutar de la majestuosidad y la sensibilidad de la poeta.

\section{CONCLUSIONES}

La complejidad estilística y temática característica de la obra de Anne Sexton, tras nuestra labor de documentación biográfica, no resulta limitante a la hora del trasvase interlingüístico del poema, gracias a la trasparencia del género confesional al que pertenece. La concatenación de metáforas, símiles y metonimias ha conseguido que el ritmo no se pierda en el Texto Meta (TM), y la nominalización, junto a una recurrente adjetivación gracias a la trasposición, nos ha otorgado las herramientas para subsanar las diferencias entre ambos idiomas. El acercamiento pragmático y los estudios precedentes en la traducción de la metáfora, desde esta perspectiva, son la clave para la obtención de una traducción independiente del texto original, que no necesite la edición en espejo en la que esté acompañada de él y que, por ende, sea más funcional. Ahí donde la diferencia cultural y los juegos de palabras han imposibilitado una traducción literal, la transcreación ha sido nuestra estrategia principal para recuperar el efecto y ha servido también como una postura conciliadora en la relación forma-contenido del TO y la forma-contenido a la que hemos recurrido en el TM.

Para finalizar, consideramos que con la propuesta de traducción que integra este estudio, el lector hispanohablante accede al potencial de la autora sin ser privado de aquellos matices cuya pérdida se nos hace irremediable. Anne Sexton juega en espejos con la imagen pública y social, el rol madrehija que nunca abandonamos, viaja de la razón al desaliento y nos abre las puertas de su casa para demostrar que lo peculiar de su salud mental no impide que nos veamos reflejados en sus versos.

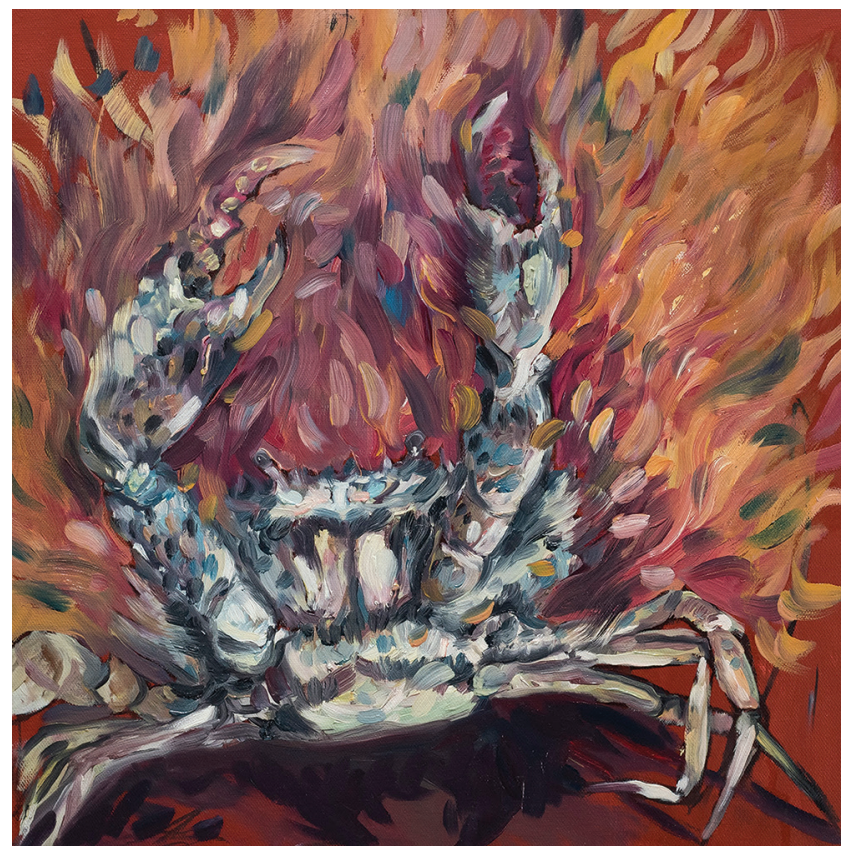

Crustáceo de agua dulce bailando alrededor del fuego, de la serie Mar incendiado (2021). Óleo sobre lino: Xilberto Loera-Núñez. Prohibida su reproducción en obras derivadas. 


\section{REFERENCIAS}

Anguita Martínez, Víctor (2019), "Los efectos secundarios del electroshock en la poesía de Anne Sexton: 'Music Swims Back to Me' y su traducción al español", Hikma, vol. 18, núm. 1, pp. 211-230, disponible en: https://doi. org/10.21071/hikma.v18i1.11207

Anguita Martínez, Víctor (2020a), Anne Sexton y Alda Merini: la creación literaria para la desinstitucionalización psiquiátrica. Traducción, poesía y relevancia, Madrid, Sindéresis.

Anguita Martínez, Víctor (2020b), "Wanting to Die de Anne Sexton y sus traducciones al español: evaluación y propuesta de traducción”, en Ma - Luisa Rodríguez y Carmen Expósito (eds.), La traducción literaria en contexto: aspectos sociales y autoría, Córdoba, UCOPress, pp. 29-51.

Etkind, Efim (1982), Un art en crise. Essai de poétique de la traduction poétique, Lausanne, L'Âge d'Homme.

Fouces González, Covadonga G. (2007), "Tras las huellas de la metáfora: una aproximación a la traducción de la metáfora literaria desde presupuestos culturales", Hermēneus. Revista de Traducción e Interpretación, num. 9, pp. 1-16, disponible en: https://recyt.fecyt.es/index.php/HS/article/ view/6222/11086

Frankl, Viktor E. (2012), Logoterapia y análisis existencial. Textos de cinco décadas, Barcelona, Herder.

Gamoneda Lanza, Amelia (2008), "La lengua bífida de la traducción”, en Javier Gómez Montero (coord.), Nuevas pautas de la traducción literaria. Cuadernos del taller de traducción literaria de Kiel 2008, Madrid, Visor libros, pp. 37-74.

Gray, Linda (2015), Anne Sexton: Un autorretrato en cartas, Ourense, Ediciones Linteo.

Helle, Anita (2016), "Anne Sexton's Photographic SelfFashioning", en Amanda Golden (ed.), This Business of Words. Reassessing Anne Sexton, Florida, University Press of Florida, pp. 38-72.

Javadizadeh, Kamran (2016), "Anne Sexton's Institutional Voice”, en Amanda Golden (ed.), This Business of Words. Reassessing Anne Sexton, Florida, University Press of Florida, pp. 73-103.

Middlebrook, Diane (1998), Anne Sexton. Una biografía, Barcelona, Circe.

Ramírez García, Tania Jesús (2009), Traducción de la metáfora poética desde un enfoque comunicativo: metáfora lorquiana, Las Palmas de Gran Canaria, Universidad de Las Palmas de Gran Canaria/Servicio de Publicaciones/Fundación Mapfre Guanarteme.

Salvio, Paula M. (2007), Anne Sexton. Teacher of Weird Abundance, Nueva York, State University of New York Press.

Samaniego Fernández, Eva (2002), "Prescripción y descripción: la metáfora en los estudios de traducción”, Trans. Revista de Traductología, núm. 6, pp. 47-61.

Sexton, Anne (1960), To Bedlam and Part Way Back, Boston, Houghton Mifflin.

Sexton, Anne (1966), Life or Die, Boston, Houghton Mifflin.

Sexton, Anne (2013), Anne Sexton. Poesía completa, Ourense, Ediciones Linteo.
Víctor Anguita Martínez. Doctor en Traducción, Lenguas y Culturas por la Universidad de Córdoba (UCO), España. Miembro del grupo de investigación HUM887: TRADISCO, en la misma institución. Sus intereses académicos son: traducción e investigación de textos literarios (poesía), estudios contrastivos en traducción literaria entre lenguas afines y análisis traductológico de índole pragmática. Entre sus publicaciones recientes encontramos: Anne Sexton y Alda Merini: la creación literaria para la desinsitucionalización psiquiátrica. Traducción, poesía y relevancia (2020); "La peregrinación al manicomio en La Tierra Santa de Alda Merini: análisis traductológico" (en Estudios de traducción, lingüística contrastiva y didáctica, 2020); y "Wanting to Die de Anne Sexton y sus traducciones al español: evaluación y propuesta de traducción" (en La traducción literaria en contexto: aspectos sociales y autoría, 2020). 